\title{
ERRATUM
}

\section{Erratum to: The status of olfactory function and the striatal dopaminergic system in drug-induced parkinsonism}

\author{
Tommaso Bovi · Angelo Antonini - Sarah Ottaviani - Angela Antonioli • \\ Maria Paola Cecchini - Vincenzo Di Francesco - Maria Antonietta Bassetto • \\ Mauro Zamboni - Antonio Fiaschi - Giuseppe Moretto - Andrea Sbarbati • \\ Francesco Osculati $\cdot$ Michele Tinazzi
}

Published online: 31 August 2010

(C) Springer-Verlag 2010

\section{Erratum to: J Neurol}

DOI 10.1007/s00415-010-5631-3

The list of author was published in incorrect order. The correct author group is given here.

The online version of the original article can be found under doi:10.1007/s00415-010-5631-3.

T. Bovi $(\bowtie) \cdot$ A. Fiaschi $\cdot$ M. Tinazzi

Dipartimento di Scienze Neurologiche e della Visione,

Università di Verona, Verona, Italy

e-mail: doctorbox@hotmail.it

A. Antonini

IRCCS San Camillo, Venice, Italy

S. Ottaviani - G. Moretto - M. Tinazzi

Unità Operativa di Neurologia OCM Borgo Trento, Verona, Italy

A. Antonioli · V. Di Francesco · M. Zamboni

Unità Operativa di Geriatria OCM Borgo Trento, Verona, Italy

M. P. Cecchini - M. A. Bassetto - A. Sbarbati

Istituto di Anatomia, Università di Verona, Verona, Italy

F. Osculati

IRCCS Centro Neurolesi “Bonino Pulejo”, Messina, Italy 